\title{
(Bio-)Synthesis of the Aquatic Phytotoxin Cyanobacterin - A Para- digm for Furanolide Core Structure Assembly
}

\author{
Paul M. D’Agostino, ${ }^{1,2, \$}$ Catharina J. Seel, ${ }^{3,+\dagger}$ Tanja Gulder, ${ }^{3,4, *}$ and Tobias A. M. Gulder ${ }^{1,2, *}$ \\ ${ }^{1}$ Technische Universität Dresden, Chair of Technical Biochemistry, Bergstraße 66, 01069 Dresden, Germany. \\ ${ }^{2}$ Biosystems Chemistry, Department of Chemistry and Center for Integrated Protein Science Munich (CIPSM), Technische \\ Universität München, Lichtenbergstraße 4, 85748 Garching bei München, Germany. \\ ${ }^{3}$ Leipzig University, Chair of Organic Chemistry, Johannisallee 29, 04103 Leipzig, Germany. \\ ${ }^{4}$ Biomimetic Catalysis, Department of Chemistry and Catalysis Research Center (CRC), Technische Universität München, \\ Lichtenbergstraße 4, 85748 Garching bei München, Germany.
}

\section{KEYWORDS: cyanobacteria, cyanobacterin, biosynthesis, furanolides, furanolide synthase, DiPaC}

\begin{abstract}
The $\gamma$-butyrolactone structural motif is commonly found in many natural signaling molecules and other specialized metabolites. A prominent example is the potent aquatic phytotoxin cyanobacterin bearing a highly functionalized $\gamma$-butyrolactone core structure. The enzymatic machinery assembling cyanobacterin and the many structurally related natural products - herein termed furanolides - has remained elusive over decades. Here we discover and characterize the underlying biosynthetic process of furanolide core structure assembly. The cyanobacterin biosynthetic gene cluster $(c y b)$ is identified by targeted bioinformatic screening and validated by heterologous expression in $E$. coli. Functional evaluation of the recombinant key enzymes provides in-depth mechanistic insights into a streamlined $C, C$-bond-forming cascade that involves installation of compatible reactivity at seemingly unreactive $\mathrm{C}-\alpha$-positions of the amino acid precursors and facilitates development of a one-pot biocatalytic in vitro synthesis. Our work extends the biosynthetic and biocatalytic toolbox for $\gamma$-butyrolactone formation. It thereby provides a general paradigm for the biosynthesis of furanolides and thus sets the stage for their targeted discovery, biosynthetic engineering and enzymatic synthesis.
\end{abstract}

Cyanobacterin (1) was the first chlorinated natural product identified from freshwater cyanobacteria when it was isolated from Scytonema hofmanni in 1982 (Figure 1). ${ }^{1}$ Its full chemical structure and stereochemistry was established in 1986 including X-ray structure analysis. ${ }^{2,3}$ It was shown to act as an inhibitor of photosynthetic electron transport in photosystem II (PSII) ${ }^{4}$ and to likewise be active against higher plants (angiosperms). ${ }^{5}$ Compared to other PSII electron transport inhibitors, such as DCMU (3-(3,4-dichlorophenyl)-1,1-dimethylurea), the minimum concentration of 1 to inhibit electron transport (inhibition of the silicomolybdate reduction) was significantly lower $\left(\mathrm{IC}_{50}=16 \mathrm{nM}\right.$ versus $300 \mathrm{nM}$ for DCMU). Cyanobacterin (1) thereby seems to target a different binding site than DCMU, since DCMU-resistant mutants are still sensitive to 1. ${ }^{6,7}$ Interestingly, the anhydro isomer ${ }^{3} \mathbf{2}$ resulting from dehydratation of $\mathbf{1}$, lacks this biological activity. ${ }^{4}$ Owing to its strong biological activity, $\mathbf{1}$ is a promising lead structure for the development of potent phytotoxins, for example, for agrochemical applications.

Cyanobacterin (1) contains a highly functionalized $\gamma$ butyrolactone core, a structural feature found in many other natural products of diverse origin, ${ }^{8}$ herein termed the furanolide natural product family. Examples include the cytotoxic nostoclide I (3) from a cyanobacterial lichen symbiont ${ }^{9}$ and the antibiotics enhygrolide ${ }^{10}(\mathbf{4})$ and angiolactone ${ }^{11}(\mathbf{5})$ from myxobacteria. The unifying structural feature of these furanolides is a $\gamma$-butyrolactone / furan-2(5H)-one core, in which substituents $\mathrm{R}^{1}(\alpha)$ and $\mathrm{R}^{2}(\beta)$ are either aromatic or aliphatic and $R^{3}(\gamma)$ exclusively aromatic (cf. common core structure 6; Figure 1, box).

Despite the pronounced biological activities and unusual structures of 1 and other furanolides, the biosynthetic process forging these compounds has remained elusive over decades. This points at unusual biosynthetic logic to be involved in furanolide assembly, which is not yet readily predictable by bioinformatics. The substitution pattern of the furanolide core 6 could indicate its assembly from three amino acid precursors. However, these would require to be condensed via the three former amino acid $C$ - $\alpha$-positions (colored dots in $\mathbf{6}$, Figure 1) that lack the required reactivity for $C, C$-bond formation (between $C-\alpha / C-\beta$ and $C-\beta /-C-\gamma$ in 6) to deliver the furanolide core structure. A new enzymatic strategy enabling the fusion of three amino acid building blocks by installing compatible $\mathrm{C}$ - $\alpha$-reactivity would thus need to be involved. We therefore set out to illuminate furanolide biosynthesis by the targeted discovery and characterization of the biosynthetic gene cluster (BGC) encoding 1. Overall, this led to a general paradigm for furanolide biosynthesis and the discovery of unprecedented $C, C$-bond forming biochemistry facilitated by the first bona fide Morita-Baylis-Hillman $(\mathrm{MBH})$ reaction catalyzing biosynthetic enzyme. 

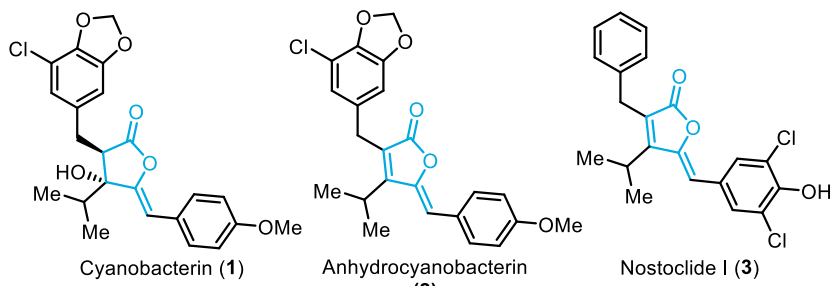

(2)
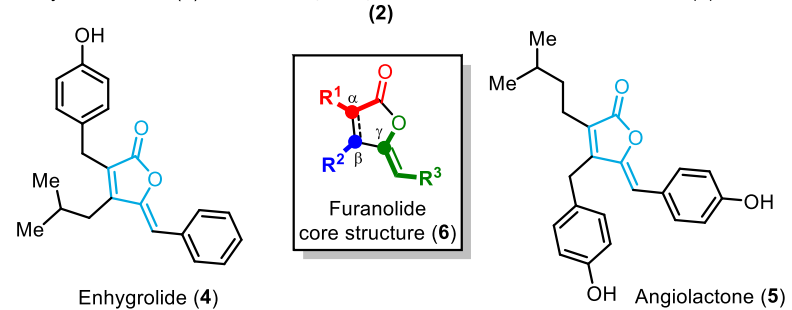

Enhygrolide (4)
Figure 1. Structures of cyanobacterin (1), its anhydro analog $\mathbf{2}$, a selection of related furanolide natural products - nostoclide I (3), enhygrolide (4), angiolactone (5) - and the common furanolide core structure (6). Former $\mathrm{C}$ - $\alpha$-positions of putative amino acid precursor are indicated by colored dots.

\section{Identification and functional validation of the cyanobacte- rin BGC (cyb)}

The reported producer of cyanobacterin (1), Scytonema hofmanni UTEX B 2349 (now referred to as Tolypothrix sp. PCC 9009), was purchased from the UTEX Culture Collection of algae and production of $\mathbf{1}$ was confirmed by LCMS analysis (Figure S1). Considering the structure of $\mathbf{1}$, which bears a chlorine substituent, the genome of Tolypothrix sp. PCC 9009 was screened for halogenase-encoding genes. A gene $c y b I$, putatively encoding a flavin-dependent halogenase (previously incorrectly annotated as a dehydrogenase), was indeed identified. Based on sequence alignment, the corresponding protein contained all known conserved residues required for halogenation (Figure S2). ${ }^{12}$ In addition, genes flanking cybI encoded functions potentially involved in cyanobacterin biosynthesis, including tyrosine metabolic proteins (a potential building block) and two $O$-methyltransferases (Table S1). The cyanobacterin BGC $(c y b)$ was predicted to be $15.7 \mathrm{~kb}$ in size and to consist of 11 genes spanning $c y b A-c y b K$ (Figure 2A). To validate its function, $c y b$ was cloned into a GFP-reporter expression vector pET28-ptetO-GFP ${ }^{13}$ in its native form as a single PCR product using Direct Pathway Cloning ( $\mathrm{DiPaC})^{13-15}$ to give vector pET28-ptetO-GFP::cyb. This vector was further refactored by removing two putative transcriptional terminators up- and downstream of $c y b I$ and all non-coding intergenic regions, resulting in vector pET28-ptetO::refcyb (Figure S3).

The refactored construct pET28b-ptetO::ref $c y b$ was heterologously expressed in E. coli $\mathrm{BAP} 1^{16}$ under the control of the tetracycline inducible ptetO promoter. A total of 4 new products not detectable in the empty-vector control experiments were identified (Figure 2B). HR-LCMS (ESI ${ }^{+}$) analysis revealed $\mathrm{m} / \mathrm{z}$ values $\left([\mathrm{M}+\mathrm{H}]^{+}\right)$of 337.1433 (compounds 7), 351.1590 (8), and $321.17(*)^{17}$ (Figure S4). The detected masses were thus significantly smaller than that of $\mathbf{1}$. Compounds $7(11.75$ and $26 \mathrm{mg} / \mathrm{L})$ and $\mathbf{8}(2.75 \mathrm{mg} / \mathrm{L})$ were produced in high amounts and thus amenable to structure elucidation by NMR (Tables S2 and S3). All products were shown to be new compounds resembling the anhydro cyanobacterin analog 2 , but only bearing $p$-phenolic functions at the aromatic substituents without further decoration (Figure 2C).
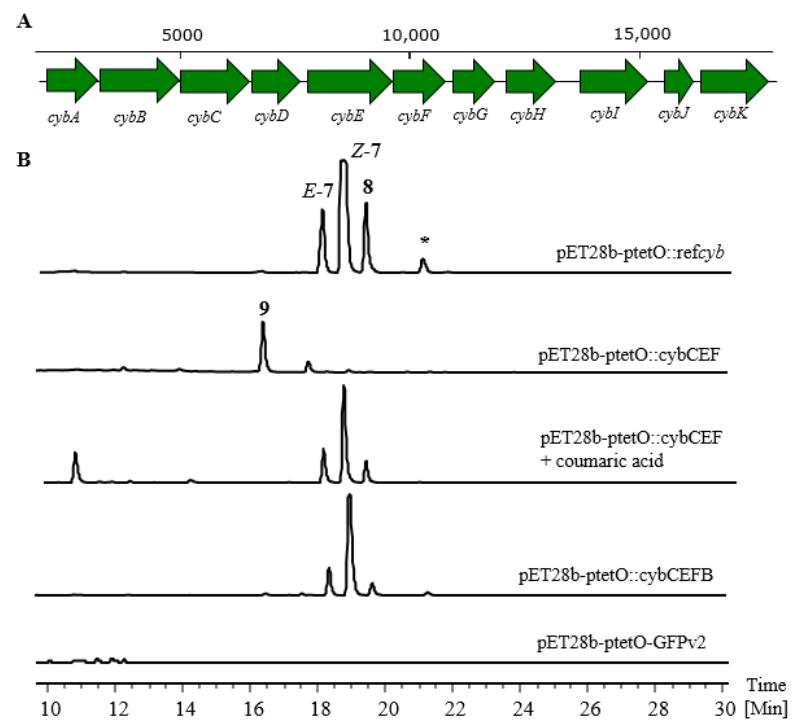

C
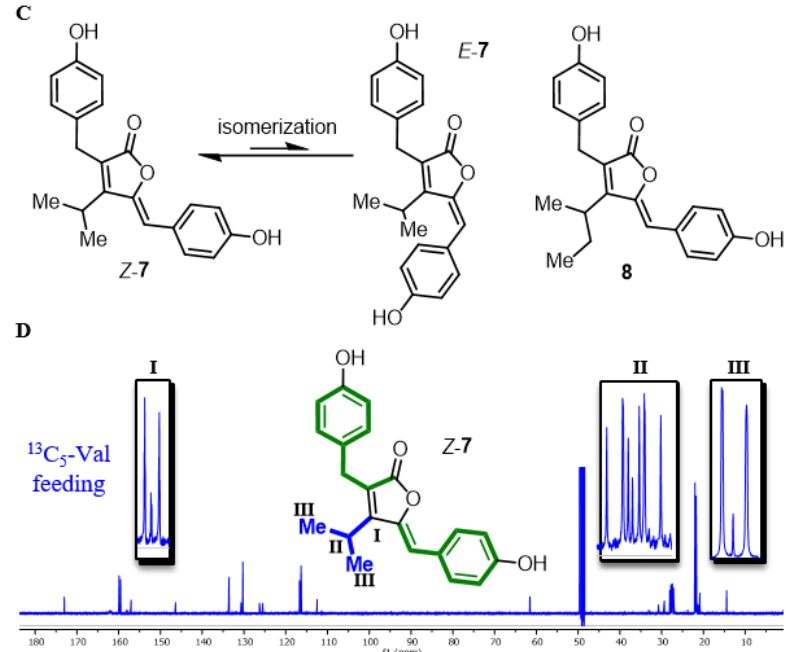

Figure 2. A. Schematic representation of $c y b$. B. HPLC-UV $(350 \mathrm{~nm})$ analysis of recombinant expression experiments with vector pET28b-ptetO-GFP containing (from top to bottom): the streamlined, complete $c y b$ BGC; $c y b C E F$; $c y b C E F$ with supplementation of coumaric acid (10); cybCEFB; empty vector. C. Structure of the isolated furanolide expression products precyanobacterins I (7) and II (8). D. Stable-isotope labelling experiment with uniformly ${ }^{13} \mathrm{C}$-labelled L-tyrosine (resulting in labelling of $\mathbf{7}$ depicted in green) or L-valine (blue). ${ }^{13} \mathrm{C}$ NMR spectrum of $\mathbf{7}$ from labelling with $\mathrm{L}$-valine is shown exemplarily (cf. Figures S6 and S7).

The metabolite pair E/Z-7 (termed precyanobacterin I) arises from $E / Z$-isomerization of the double bond located at the $\gamma$ position of the furanolide core. This resembles previous observations of other known furanolides such as enhygrolide (4) and anhydro-cyanobacterin 2 (Figure 1). ${ }^{3,10}$ The $E$-isomer $E$-7 seems to be the direct biosynthetic product, which slowly interconverts into the thermodynamically preferred $Z$-isomer Z-7 in solution (Figure S5). Compound 8 (precyanobacterin II), with a 14 u higher molecular mass when compared to 7 , was shown to possess a 2-butane substituent at $\beta$-position of the furanolide core (Table S3). 
Establishing the building blocks and the minimal BGC for furanolide core biosynthesis

While the established expression system did not yield cyanobacterin (1) itself, indicating the required tailoring steps not to be functional, it paved the way to elucidate the enigmatic $C, C$ bond formation in furanolide core structure assembly. To get first insights into the required biosynthetic building blocks, stable-isotope labelling experiments were performed using the potential precusors L-tyrosine and L-valine. Feeding these amino acids in a uniformly ${ }^{13} \mathrm{C}$-labelled form in two separate experiments indeed revealed all carbon atoms in 7 to be derived of these precursors (Figure 2D, Figures S6 and S7). The furanolide core is thus exclusively assembled from amino acid building blocks, despite being devoid of nitrogen. Most importantly, the $C, C$-bond forming events required to establish the central furanolide core structure must occur between the three former $\alpha$-positions of these amino acids (cf. Figure 1, colored dots in the structure of 7), pointing at the involvement of unprecedented biochemistry.

To identify the minimal set of genes required for core structure assembly, we applied a gene knockout approach utilizing DiPaC and SLIC DNA assembly (Figure S8). ${ }^{15}$ Based on the predicted function of the proteins encoded within $c y b$, we initially excluded elements putatively involved in amino acid metabolism and tailoring reactions (i.e., genes encoding CybA = DHAP synthase; $\mathrm{CybB}=$ aromatic amino acid ammonia lyase; $\mathrm{CybD}=O$-methyltransferase $; \mathrm{CybH}=$ phenylpropionate dioxygenase; $\mathrm{CybI}=$ halogenase $; \mathrm{CybJ}=O$-methyltransferase; CybK = tyrosinase; cf. Table S1) and rather concentrated our efforts on the genes encoding CybC (long-chain acyl-CoA synthetase), CybE (TPP-binding protein), CybF (3-oxoacyl[acyl-carrier-protein]-synthase, and CybG (uncharacterized protein with $\mathrm{NAD}(\mathrm{P})$ binding site). Of particular interest was $\mathrm{CybE}$, as TPP-dependent enzymes are involved in many biosynthetic Umpolung reactions, suggesting a major role in $C, C$ bond construction. ${ }^{18,19}$ While deletion of $c y b G$ within pET28ptetO::ref $c y b$ did not change the metabolic profile, knock-outs of either $c y b C, c y b E$ or $c y b F$ resulted in complete abolishment of furanolide production (Figure S9). We thus cloned a minimal expression construct exclusively containing these three genes, pET28-ptetO::refcybCEF. While experiments with this vector again did not show formation of any furanolide, the new product 9 was observed (cf. Figures 2B and 3). NMR analysis of 9 isolated from expression experiments with uniformly ${ }^{13} \mathrm{C}$-labeled amino acid building blocks - applied both, individually and in a single experiment - permitted its structural assignment as acyloin 9 (Figure S10), a putative biosynthetic intermediate or shunt product. Comparison of the structures of 7 and 9 revealed a $p$-coumaric acid (10) unit missing for core structure assembly. Indeed, supplementation of 4coumaric acid (10) to recombinant expression experiments with pET28-ptetO::ref $c y b C E F$ fully restored furanolide production (Figure 2B). Re-inspection of $c y b$ suggested $c y b B$, encoding an ammonia lyase, might be required to provide $\mathbf{1 0}$ from L-tyrosine. ${ }^{20,21}$ Insertion of $c y b B$ into the pET28ptetO::ref $c y b C E F$ expression vector delivering pET28ptetO::ref $c y b C E F B$ finally resulted in identical results when compared to pET28-ptetO::ref cyb expression (Figure 2B), thus establishing the minimal pathway required for furanolide assembly in vivo to consist of only four genes, $c y b B C E F$.
Early steps of furanolide core structure assembly - the catalytic functions of $\mathrm{CybB}, \mathrm{CybC}$, and $\mathrm{CybE}$

The conducted stable-isotope labelling, gene knock-out, chemical and genetic complementation experiments delivered a conclusive picture of the early steps of furanolide biosynthesis. The TPP-dependent enzyme CybE thereby catalyzes formation of the observed acyloin 9. CybE thus acts identical to homologous TPP-dependent enzymes characterized in various natural product biosynthetic pathways, including those of scytonemin (ScyA), ${ }^{19,22}$ sattabacin (Thz0150) ${ }^{23}$ and sattazolin (Cbei2730). ${ }^{24}$ These enzymes employ diverse $\alpha$-keto acid analogs to perform an acyloin coupling, resulting in $C, C$-bond formation to initially deliver an $\alpha$-hydroxy- $\beta$-ketoacid product. While in case of scytonemin biosynthesis, two distinct dehydrogenases provide each $\alpha$-keto acid substrate, the $c y b$ pathway does not encode such enzymatic function. However, the required keto acid precursors of CybE are ubiquitous intermediates in amino acid metabolism and can thus be provided by house-keeping transaminases (TA) in both, Tolypothrix sp. PCC 9009 and the recombinant E. coli expression host. CybE performs acyloin coupling utilizing 4-hydroxyphenylpyruvic acid (11, derived of L-tyrosine) and $\alpha$-ketoisovaleric acid (12, derived of L-valine) (Figure 3A). Pyruvic acid 11 gets attached to the TPP-ylide cofactor by nucleophilic attack to give 13, which upon decarboxylation delivers 14. Nucleophilic attack of enamine $\mathbf{1 4}$ at the keto function in $\mathbf{1 2}$ furnishes $\mathbf{1 5}$, which gets released from the enzyme to form carboxylic acid $\mathbf{1 6}$ in analogy to previous reports of homologous enzymes. ${ }^{19,22,24}$ Spontaneous decarboxylation leads to 9 , the compound observed in the recombinant expression experiments with CybCEF (cf. Figure 2). This likely is a biosynthetic shunt product, with 16 being the true biosynthetic intermediate required for furanolide assembly (cf. Figure 4). Overall, CybE thus connects the first two building blocks derived of primary metabolism, thereby establishing the $C, C$-bond between the $\alpha$ and $\beta$-position in the furanolide core (cf. Figure 1, box).

As shown by the chemical complementation experiments above, the second required molecular building block for furanolide biosynthesis is 4-coumaric acid (10), which is provided by $\mathrm{CybB}$ by elimination of ammonia from L-tyrosine (Figure 3). CybC, annotated as long-chain acyl-CoAsynthetase, was found to have high similarity with the 4coumarate:coenzyme A ligase (4CL) family of enzymes, which convert various cinnamic acids to their respective CoA esters. $^{25,26} \mathrm{CybC}$ will thus likely utilize $\mathbf{1 0}$ to give activated CoA ester 17. CybB and CybC thus convert L-tyrosine to 4coumaroyl-CoA (17), identical to the initial steps of stilbene biosynthesis (StlA and StlB). ${ }^{27}$ Overall, CybB, CybC and CybE provide the two molecular building blocks that are fused to form the furanolide core by a single enzyme, CybF. 


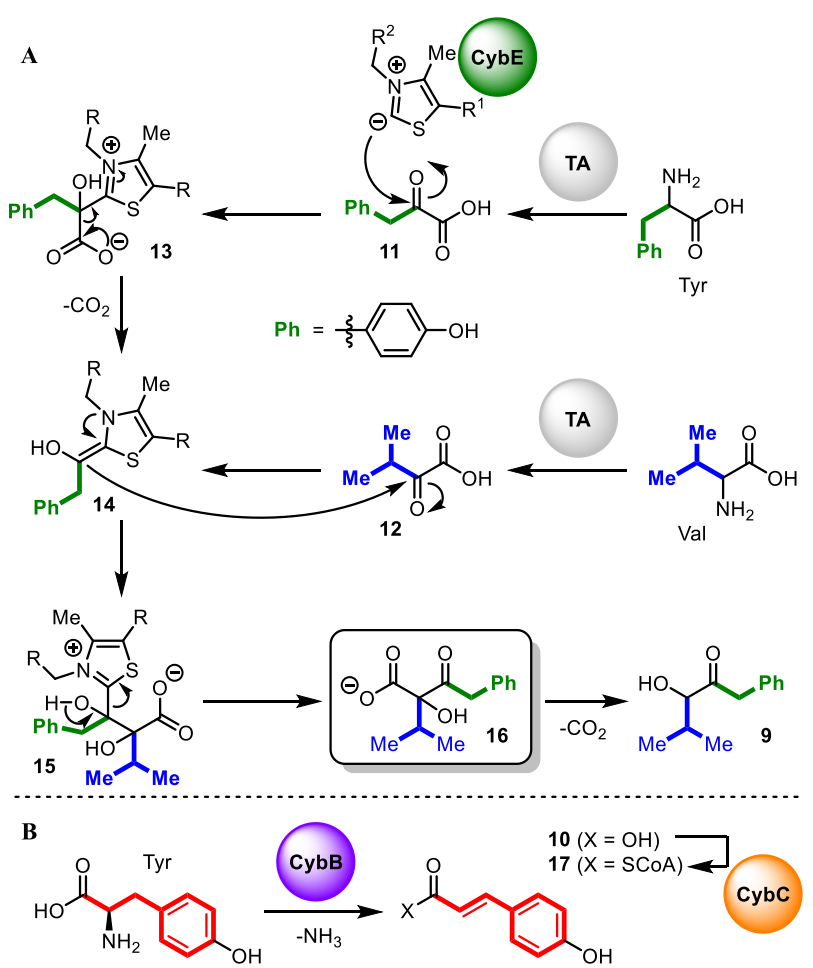

Figure 3. A. Assembly of $\mathbf{1 6}$ from 11 and $\mathbf{1 2}$ derived of Ltyrosine and L-valine (provided by transaminases (TA) from primary metabolism), respectively, by the TPP-dependent enzyme CybE. $R^{1}$ and $R^{2}$ in the depicted TPP cofactor are omitted for clarity $\left(\mathrm{R}^{1}=\right.$-ethyldiphosphate, $\mathrm{R}^{2}=-$ pyrimidine2,4-diamine). B. Biosynthesis of 4-coumaroyl-CoA (17) catalyzed by $\mathrm{CybB}$ and $\mathrm{CybC}$.

CybF catalyzes an unprecedented acylation / C, $C$-bond formation / hydride shift reaction cascade

The last enzyme proven obligatory for furanolide core assembly by the recombinant expression experiments was $\mathrm{CybF}$. This suggests it to catalyze a remarkable reaction cascade that efficiently fuses 16 and 4-coumaroyl-CoA (17). CybF is annotated as a 3-oxoacyl-[ACP] synthase containing a FabH domain. These constitute a large family of fatty acid and natural product biosynthetic enzymes that catalyze a range of highly diverse reactions. ${ }^{28}$ Based on the available substrates for $\mathrm{CybF}$, compounds 16 and 17, we propose an initial decarboxylative $O$-acylation to give $\mathbf{1 8}$ (Figure 4A). Subsequent tethering of $\mathbf{1 8}$ to $\mathrm{CybF}$ by nucleophilic attack of an active-site residue onto the $p$-coumaric acid-derived Michael system gives enolate 19. This sets the stage for $C, C$-bond formation to $\mathbf{2 0}$, followed by detachment of intermediate $\mathbf{2 1}$ from the enzyme. This process resembles a Morita-Baylis-Hillman $(\mathrm{MBH})$ reaction mechanism and thus defines $\mathrm{CybF}$ as the first bona fide $\mathrm{MBH}-$ catalyzing enzyme. ${ }^{29-31}$ While the furanolide carbon framework is thus fully established, positioning of the double-bonds in 21 does not correspond to that observed in the final product 7. Adjustment of these structural features needs to take place in the absence of an additional Red/Ox-enzyme. We therefore suggest a unique 1,4-hydride shift to install the required exocyclic double bond in $\mathbf{2 2}$ (located at the molecular portion depicted in green), concomitantly removing the double-bond at the former $p$-coumaric acid residue. This hydride-shift directly delivers enolate $\mathbf{2 2}$, which is set up for elimination of $\mathrm{H}_{2} \mathrm{O}$ to ultimately give precyanobacterin I (7).

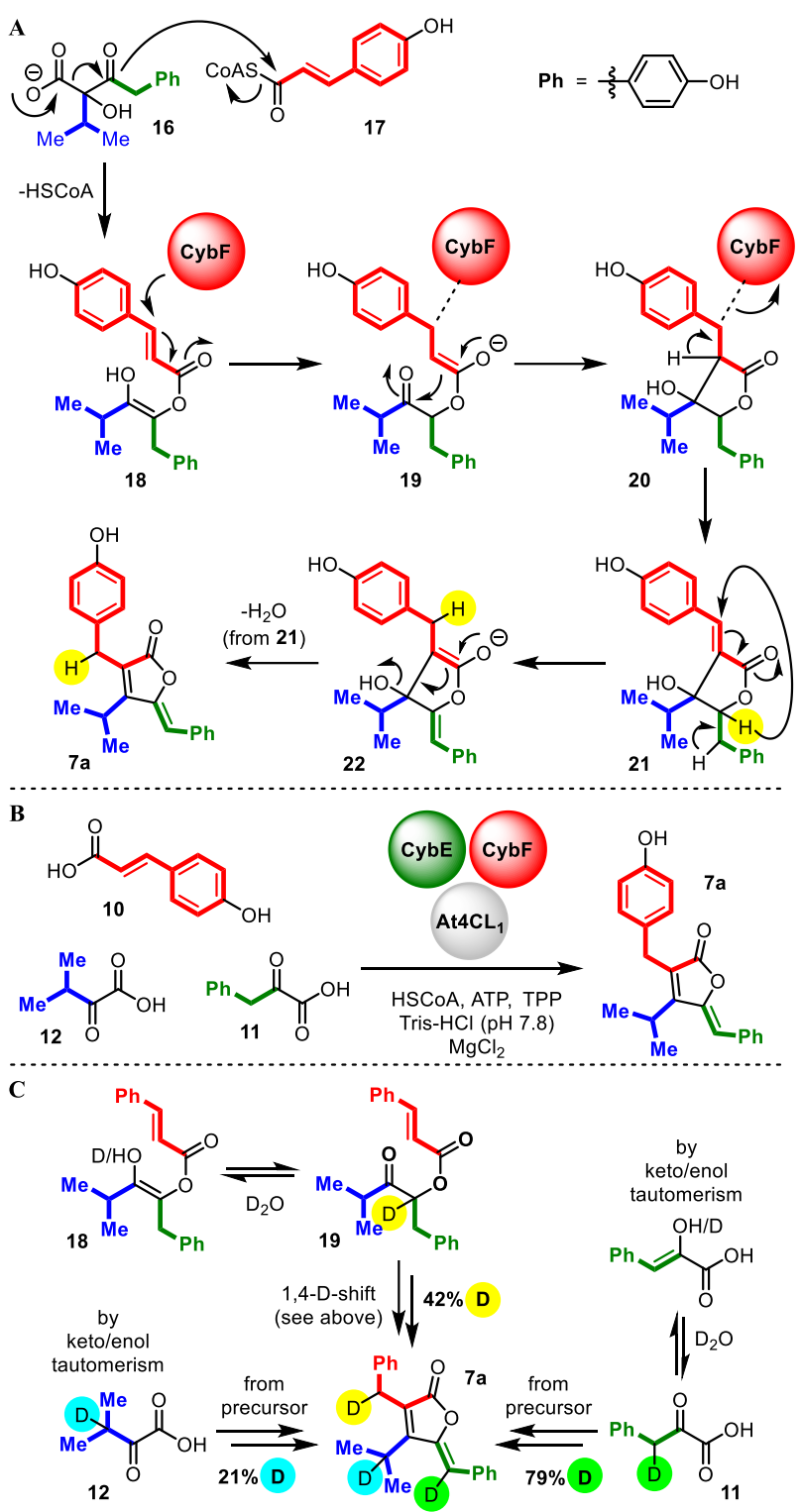

Figure 4. A. Proposed reaction course of CybF-catalyzed assembly of the furanolide core from the two intermediates $\mathbf{1 6}$ and $\mathbf{1 7}$ involving an acylation / $C, C$-bond formation / hydride shift reaction cascade. B. Biocatalytic total synthesis of precyanobacterin I (7). C. In-vitro verification of the final 1,4hydride shift reaction that facilitates adjustment of the oxidative state from $\mathbf{2 1}$ to $\mathbf{7}$ without involvement of an additional Red/Ox-enzyme. The reaction was carried out in $\mathrm{D}_{2} \mathrm{O}$, leading to deuterium incorporation at three positions, at two of which by keto/enol-tautomerism (blue and green dots) and one by 1,4-deuterium-shift after core structure cyclization (yellow).

\section{Enzymatic synthesis of precyanobacterin I (7) - functional} validation of key biosynthetic steps

To enable further experimental validation of the proposed pathway, we next individually cloned $c y b C, c y b E$, and $c y b F$ into a pHis8-TEV vector for recombinant expression of the respective fusion proteins in E. coli (see SI, Figure S11). This resulted in sufficient amounts of soluble $\mathrm{CybC}, \mathrm{CybE}$ and $\mathrm{CybF}$ for in vitro application. However, CybC turned out to be inactive in the performed assays. It was thus replaced by the homologous enzyme At4CL1 from Arabidopsis thaliana, 
which was successfully overexpressed from a pET30a(+) vector delivering $\mathrm{His}_{6}$-At4CL1. ${ }^{26}$ Application of these enzymes in vitro together with the CybE substrates 4hydroxyphenylpyruvic acid (11) and $\alpha$-ketoisovaleric acid (12), $p$-coumaric acid (10), HSCoA, ATP and $\mathrm{Mg}^{2+}$ (see SI for details) indeed led to successful and selective production of 7 , thereby establishing a first, fully biocatalytic total synthesis of the furanolide core structure (Figure 4B).

This in vitro setup was furthermore harnessed to investigate the proposed reaction sequence of $\mathrm{CybF}$, in particular the 1,4hydride shift reaction. We reasoned that performing the biocatalytic synthesis of 7 in $\mathrm{D}_{2} \mathrm{O}$ would be suitable to experimentally prove this transfer. Two positions in 7 were thought to be deuterated due to keto/enol tautomerization leading to mono-deuterium-labelling of the 4-hydroxyphenylpyruvic acid (11, corresponding deuterium highlighted in green) and $\alpha$ ketoisovaleric acid (12, blue) precursors. The assay was conducted in sufficiently large scale to permit subsequent isolation and NMR analysis of generated 7 (SI, Figure S12). This indeed revealed that both positions in 7 were deuterated, as expected to a significantly larger extent at the position derived of 11 (approx. 79\%) versus that from 12 (approx. 21\%), as a result of the higher acidity of the corresponding position in $\mathbf{1 1}$ (Figure 4C). Additionally, deuteration was thought possible in pathway intermediate 19, prior to CybE-catalyzed $C, C$-bond formation to $\mathbf{2 0}$. If the proposed 1,4-hydride shift indeed occurred, this labelling would be transferred to the Michael acceptor of the former $p$-coumaric acid residue in 21 (cf. Figure $4 \mathrm{~A}$, highlighted in yellow). To our delight, significant deuterium transfer was indeed observed (42\%), providing unambiguous direct evidence for the postulated 1,4-hydride shift in the assembly of 7, as well as indirect evidence for the existence of $\mathbf{1 8 / 1 9}$ as intermediates and hence for the entire proposed sequence catalyzed by $\mathrm{CybF}$. Given the unprecedented reaction cascade catalyzed by $\mathrm{CybF}$ and its decisive role in furanolide core structure assembly, we designate this enzyme as furanolide synthase (FuSy).

\section{Conclusions}

Within this work, we establish a biosynthetic paradigm for the assembly of the furanolide core structure 6. Initial steps of the pathway resemble stilbene (i.e., production of 4-coumaroylCoA (17) by $\mathrm{CybB}$ and $\mathrm{CybC}$ ) and acyloin natural product biosynthesis (i.e., formation of intermediate 19 by CybE). Ultimately, the provided precursors are fused by the FuSy $\mathrm{CybF}$, which catalyzes an unprecedented reaction cascade combining a formal $\mathrm{MBH}$ reaction and a final 1,4-hydrideshift that facilitates late-stage adjustment of oxidative states at residues $R^{3}$ and $R^{1}$ without involvement of a redox cofactor. While enzymatic MBH reactions have been reported as applications of side-reactions of promiscuous nucleophilic carrier proteins, such as albumins or hydrolytic enzymes, ${ }^{32-34} \mathrm{CybF}$ constitutes the first bona fide $\mathrm{MBH}$-catalyzing enzyme and thus significantly expands the natural biocatalytic repertoire for $C, C$-bond formation. Overall, this remarkable biosynthetic process fuses three amino acids by $C, C$-bond installation between all former $\mathrm{C}$ - $\alpha$-positions and thus delivers the nitrogenfree furanolide structural framework. Our work sets the stage for the discovery of the family of furanolide BGCs and related biosynthetic pathways employing CybF homologs, now possible by targeted genome mining. It also forms the basis for the genetic engineering of furanolide BGCs and the biocatalytic application of the discovered enzymatic machinery for the design and optimization of furanolide-type bioactive small molecules with potential applications in medicine and agrochemistry. This work, as well as the elucidation of the molecular mechanistic details of the CybF reaction cascade, are currently pursued in our laboratories.

\section{Online content}

Supporting information: materials and methods, strain and plasmid lists, NMR spectra and plasmid maps.

\section{Acknowledgements}

We would like to thank Prof. Clint Chapple (Purdue University) and Dr. Peng Wang (Willow Biosciences) for providing the pET30a(+)::At4CL1 vector. We also thank Dr. Lara Wolf and Prof. Dr. Weuster Botz (Technical University of Munich) for technical support.

P.M.D. thanks the Marie Skłodowska-Curie Actions Individual Fellowship (Project ID: 745435) for funding. C.J.S thanks the DBU fellowship (grant 20015/400). Research in the T.A.M.G. (DFG GU 1233/1-1) and T.G. (DFG GU 1134/4-1) laboratories is generously funded by the German Research Foundation.

\section{Author Contributions}

The manuscript was written through contributions of all authors. All authors have given approval to the final version of the manuscript. ${ }^{\top}$ These authors contributed equally.

\section{Competing interests}

The authors declare no competing interests

\section{Additional information}

Correspondence and requests for materials should be addressed to T.G. or T.A.M.G.

\section{Corresponding Authors}

* T.G.: tanja.gulder@uni-leipzig.de. T.A.M.G.: tobias.gulder@tu-dresden.de.

\section{Present Addresses}

†Currenta GmbH \& Co. OHG, CHEMPARK, 51368 Leverkusen; Catharinajulia.seel@ currenta.biz

\section{OrcID}

P.M.D: 0000-0002-8323-5416.

T.G: 0000-0003-4870-2266.

TAMG: 0000-0001-6013-3161. 


\section{References}

1. Mason, C. P. et al. Isolation of chlorine-containing antibiotic from the freshwater cyanobacterium Scytonema hofmanni. Science 215, 400 (1982).

2. Gleason, F. K., Porwoll, J., Flippen-Anderson, J. L. \& George, C. X-ray structure determination of the naturally occurring isomer of cyanobacterin. J. Org. Chem. 51, 1615-1616 (1986).

3. Pignatello, J. J. et al. Structure of the antibiotic cyanobacterin, a chlorine-containing .gamma.-lactone from the freshwater cyanobacterium Scytonema hofmanni. J. Org. Chem. 48, 4035-4038 (1983).

4. Gleason, F. K. \& Paulson, J. L. Site of action of the natural algicide, cyanobacterin, in the blue-green alga, Synechococcus sp. Arch. Microbiol. 138, 273-277 (1984).

5. Gleason, F. K. \& Case, D. E. Activity of the natural algicide, cyanobacterin, on angiosperms. Plant Physiol. 80, 834 (1986).

6. Gleason, F. K., Case, D. E., Sipprell, K. D. \& Magnuson, T. S. Effect of the natural algicide, cyanobacterin, on a herbicide-resistant mutant of Anacystis nidulans R2. Plant Sci. Amst. Neth. 46, 5-10 (1986).

7. Mallipudi, L. R. \& Gleason, F. K. Characterization of a mutant of Anacystis nidulans $\mathrm{r} 2$ resistant to the natural herbicide, cyanobacterin. Plant Sci. Amst. Neth. 60, 149-154 (1989).

8. Negishi, E. \& Kotora, M. Regio- and stereoselective synthesis of $\gamma$-alkylidenebutenolides and related compounds. Tetrahedron 53, 6707-6738 (1997).

9. Yang, X., Shimizu, Y., Steiner, J. R. \& Clardy, J. Nostoclide I and II, extracellular metabolites from a symbiotic cyanobacterium, Nostoc sp., from the lichen Peltigera canina. Tetrahedron Lett. 34, 761-764 (1993).

10. Felder, S. et al. Salimyxins and enhygrolides: Antibiotic, sponge-related metabolites from the obligate marine myxobacterium Enhygromyxa salina. Chembiochem 14, 1363-1371 (2013).

11. Raju, R., Garcia, R. \& Müller, R. Angiolactone, a new butyrolactone isolated from the terrestrial myxobacterium, Angiococcus sp. J. Antibiot. (Tokyo) 67, 725726 (2014).

12. van Pée, K.-H. \& Patallo, E. P. Flavin-dependent halogenases involved in secondary metabolism in bacteria. Appl. Microbiol. Biotechnol. 70, 631 (2006).

13. Duell, E. R. et al. Direct pathway cloning of the sodorifen biosynthetic gene cluster and recombinant generation of its product in E. coli. Microb. Cell Factories 18, 32 (2019).

14. Christian Greunke et al. Direct pathway cloning (Di$\mathrm{PaC}$ ) to unlock natural product biosynthetic potential. Metab. Eng. 47, 334-345 (2018).

15. D’Agostino, P. M. \& Gulder, T. A. M. Direct pathway cloning combined with sequence- and ligationindependent cloning for fast biosynthetic gene cluster refactoring and heterologous expression. ACS Synth. Biol. 7, 1702-1708 (2018).

16. Pfeifer, B. A., Admiraal, S. J., Gramajo, H., Cane, D. E. \& Khosla, C. Biosynthesis of complex polyketides in a metabolically engineered strain of E. coli. Science 291, 1790-1792 (2001).

17. While production levels of $(*)$ were too low for a full structure elucidation, its molecular mass suggests it to be an analog of 7 with only one phenolic $\mathrm{OH}$ group.

18. Jordan, F. Current mechanistic understanding of thiamin diphosphate-dependent enzymatic reactions. Nat. Prod. Rep. 20, 184-201 (2003).

19. Balskus, E. P. \& Walsh, C. T. Investigating the initial steps in the biosynthesis of cyanobacterial sunscreen scytonemin. J. Am. Chem. Soc. 130, 15260-15261 (2008).

20. Louie, G. V. et al. Structural determinants and modulation of substrate specificity in phenylalanine-tyrosine ammonia-lyases. Chem. Biol. 13, 1327-1338 (2006).

21. Moffitt, M. C. et al. Discovery of two cyanobacterial phenylalanine ammonia lyases: Kinetic and structural characterization. Biochemistry 46, 1004-1012 (2007).

22. Balskus, E. P. \& Walsh, C. T. An enzymatic cyclopentyl $[b]$ indole formation involved in scytonemin biosynthesis. J. Am. Chem. Soc. 131, 14648-14649 (2009).

23. Park, J.-S. et al. Identification and biosynthesis of new acyloins from the thermophilic bacterium Thermosporothrix hazakensis SK20-1 ${ }^{\mathrm{T}}$. Chembiochem 15, 527532 (2014).

24. Schieferdecker, S. et al. Biosynthesis of diverse antimicrobial and antiproliferative acyloins in anaerobic bacteria. ACS Chem. Biol. 14, 1490-1497 (2019).

25. Knobloch, K.-H. \& Hahlbrock, K. 4-coumarate: CoA ligase from cell suspension cultures of Petroselinum hortense Hoffm: Partial purification, substrate specificity, and further properties. Arch. Biochem. Biophys. 184, 237-248 (1977).

26. Li, X., Bonawitz, N. D., Weng, J.-K. \& Chapple, C. The growth reduction associated with repressed lignin biosynthesis in Arabidopsis thaliana is independent of flavonoids. Plant Cell 22, 1620 (2010).

27. Joyce, S. A. et al. Bacterial biosynthesis of a multipotent stilbene. Angew. Chem. Int. Ed. 47, 1942-1945 (2008).

28. Nofiani, R., Philmus, B., Nindita, Y. \& Mahmud, T. 3Ketoacyl-ACP synthase (KAS) III homologues and their roles in natural product biosynthesis. MedChemComm 10, 1517-1530 (2019).

29. Wei, Y. \& Shi, M. Recent advances in organocatalytic asymmetric Morita-Baylis-Hillman/aza-MoritaBaylis-Hillman reactions. Chem. Rev. 113, 6659-6690 (2013).

30. Chandra Bharadwaj, K. Intramolecular MoritaBaylis-Hillman and Rauhut-Currier reactions. A catalytic and atom economic route for carbocycles and heterocycles. RSC Adv. 5, 75923-75946 (2015).

31. Basavaiah, D. \& Naganaboina, R. T. The BaylisHillman reaction: A new continent in organic chemistry - our philosophy, vision and over three decades of research. New J. Chem. 42, 14036-14066 (2018).

32. Reetz, M. T., Mondière, R. \& Carballeira, J. D. Enzyme promiscuity: First protein-catalyzed MoritaBaylis-Hillman reaction. Tetrahedron Lett. 48, 16791681 (2007). 
33. Jiang, L. \& Yu, H. An example of enzymatic promiscuity: the Baylis-Hillman reaction catalyzed by a biotin esterase (BioH) from Escherichia coli. Biotechnol. Lett. 36, 99-103 (2014).
34. Tian, X., Zhang, S. \& Zheng, L. First Novozym 435 lipase-catalyzed Morita-Baylis-Hillman reaction in the presence of amides. Enzyme Microb. Technol. 84, 32-40 (2016). 